\title{
Delivering cognitive therapy for adolescent social anxiety disorder in NHS CAMHS: a clinical and cost analysis
}

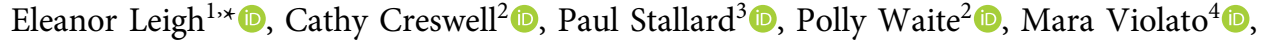 \\ Samantha Pearcey ${ }^{2}$ (D) Emma Brooks ${ }^{5}$ (D) Lucy Taylor ${ }^{6}$ (D), Emma Warnock-Parkes ${ }^{1}$ (i) and \\ David M. Clark ${ }^{1}$ (I)
}

\begin{abstract}
${ }^{1}$ Department of Experimental Psychology, University of Oxford, Oxford Centre for Anxiety Disorders and Trauma, The Old Rectory, Paradise Square, Oxford OX1 1TW, UK, ${ }^{2}$ Department of Experimental Psychology and Department of Psychiatry, University of Oxford, Anna Watts Building, Radcliffe Observatory Quarter, Woodstock Road, Oxford OX2 6GG, UK, ${ }^{3}$ Child and Adolescent Mental Health Service, Oxford Health NHS Trust, Temple House, Temple Street, Keynsham, Bristol BS31 1HA, UK, ${ }^{4}$ Health Economics Research Centre, Nuffield Department of Population Health, NIHR Oxford Biomedical Research Centre, University of Oxford, Richard Doll Building, Old Road Campus, Oxford OX3 7LF, UK, ${ }^{5}$ School of Psychology and Clinical Language Sciences, University of Reading, Earley Gate, Whiteknights, Reading RG6 6AL, UK and ${ }^{6}$ Department of Psychiatry, University of Oxford, Warneford Hospital, Oxford OX3 7JX, UK ${ }^{*}$ Corresponding author. Email: eleanor.leigh@psy.ox.ac.uk
\end{abstract}

(Received 14 February 2020; revised 6 July 2020; accepted 4 August 2020; first published online 01 March 2021)

\begin{abstract}
Background: Cognitive therapy, based on the Clark and Wells (1995) model, is a first-line treatment for adults with social anxiety disorder (SAD), and findings from research settings suggest it has promise for use with adolescents (Cognitive Therapy for Social Anxiety Disorder in Adolescents; CT-SAD-A). However, for the treatment to be suitable for delivery in routine clinical care, two questions need to be addressed. Aims: Can therapists be trained to achieve good outcomes in routine Child and Adolescent Mental Health Services (CAMHS), and what are the costs associated with training and treatment?

Method: CAMHS therapists working in two NHS trusts received training in CT-SAD-A. They delivered the treatment to adolescents with SAD during a period of supervised practice. We examined the clinical outcomes for the 12 patients treated during this period, and estimated costs associated with treatment and training.

Results: Treatment produced significant improvements in social anxiety symptoms, general anxiety and depression symptoms, and reductions in putative process measures. Seventy-five per cent (9 out of 12) patients showed a reliable and clinically significant improvement in social anxiety symptoms, and $64 \%(7 / 11)$ lost their primary diagnosis of SAD. The total cost to the NHS of the CT-SAD-A treatment was $£ 4047$ ( $S D=£ 1003$ ) per adolescent treated, of which $£ 1861(S D=£ 358)$ referred to the specific estimated cost of face-to-face delivery; the remaining cost was for training and supervising therapists who were not previously familiar with the treatment.

Conclusions: This study provides preliminary evidence that clinicians can deliver good patient outcomes for adolescents with SAD in routine CAMHS during a period of supervised practice after receiving a 2-day training workshop. Furthermore, the cost of delivering CT-SAD-A with adolescents appeared to be no more than the cost of delivering CT-SAD with adults.
\end{abstract}

Keywords: adolescents; CAMHS; CBT; psychological therapy; social anxiety disorder; young people 


\section{Introduction}

Social anxiety disorder (SAD) is common and usually first occurs during adolescence (Kessler et al., 2005; Merikangas et al., 2009), with almost all cases occurring by early adulthood (Beesdo et al., 2010). It is associated with high levels of impairment in education and work, social relationships, and day-to-day life (La Greca and Harrison, 2005; Ranta et al., 2009; Stein and Kean, 2000; Wittchen et al., 1999) and has a particularly low natural recovery rate in the absence of treatment (Bruce et al., 2005; Ranta et al., 2009). Developing effective and scalable treatments for adolescent SAD will be important to offset the individual, NHS and societal costs associated with the disorder.

Cognitive therapy for SAD (CT-SAD) is a distinctive form of cognitive behavioural therapy (CBT) that is highly effective for adults (Mayo-Wilson et al., 2014). It aims to reverse the processes specified in the cognitive model of social anxiety developed by Clark and Wells (1995). Comparisons with other active treatments for adults have demonstrated that CT-SAD is superior to: two forms of group CBT, exposure therapy, interpersonal psychotherapy, psychodynamic psychotherapy, SSRIs, medication-focused treatment as usual, and placebo medication (Clark et al., 2003; Clark et al., 2006; Leichsenring et al., 2013; Mörtberg et al., 2007; Stangier et al., 2003; Stangier et al., 2011). Individual CBT (including Clark and Wells' CT-SAD) is recommended as the first-choice treatment for adult SAD in the current NICE Guideline (NICE, 2013), and was judged to be most cost-effective in the long-term. The Guideline noted the limited data for psychological interventions in youth SAD, and concluded that CBT was the most promising intervention, and further that CT-SAD should be considered for the treatment of adolescents with social anxiety. There is currently a lack of studies examining moderators of CBT for youth SAD, although for CBT in general, from the studies that have been carried out, it seems that factors such as modality and parental involvement may not affect response (Creswell et al., 2020).

In line with this, a randomised controlled trial with adolescents (Ingul et al., 2014) demonstrated that CT-SAD was superior to both a psychological placebo and a generic form of CBT on self and clinician ratings at post-treatment. At 12-month follow-up when the two treatment arms were compared, it was found that gains and group differences persisted. The study is significant as it demonstrates treatment specificity of cognitive therapy for SAD in adolescents. Whilst the findings are notable, CT-SAD was not implemented fully in the study. Interventions for socially traumatic memories were not included, and psychoeducation and anxiety hierarchies, which are not part of CT-SAD, were used. Subsequently, Leigh and Clark (2016) undertook a pilot study to examine whether the full CT-SAD could be delivered to adolescents with appropriate age-relevant adaptation (Cognitive Therapy for Social Anxiety Disorder in Adolescents; CT-SAD-A). At the end of treatment, all participants were free of all diagnoses and this improvement was maintained at follow-up, with an average reduction of $87 \%$ on a social anxiety symptom scale. Taken together, these findings are promising and point to the potential value of delivering CT-SAD early in the course of social anxiety. However, the therapist in the pilot study had received extensive training in CT and we know from research with socially anxious adults that specific CT competencies are needed for good outcomes (Ginzburg et al., 2012). Therefore, the question arises as to whether modestly trained therapists in routine Child and Adolescent Mental Health Services (CAMHS) can also achieve good patient outcomes. This will be important to demonstrate if CT-SAD-A is to be disseminated more widely. Related to this, it is also necessary to estimate the NHS costs associated with training therapists and delivery of CT-SAD-A, as this can provide a first insight into the economic viability of the treatment in routine clinical practice.

The study had two main aims: first, to train NHS CAMHS therapists to deliver the adapted treatment and to assess young people's outcomes; and second, to estimate the cost of delivering CT-SAD-A within an NHS CAMHS setting. 


\section{Method}

\section{Ethics and consent}

The study was approved by the NHS South Central - Oxford B Research Ethics Committee (REC reference: 16/SC/0315) and University of Reading Research Ethics Committee (UREC 16/43). Informed consent/assent was sought from parents and from young people.

\section{Treatment}

CT-SAD-A, which is the adolescent version of CT-SAD for adults based on the Clark and Wells (1995) model, is a distinctive form of CBT delivered in 14 weekly 1.5 -hour sessions provided on a one-to-one basis. CT-SAD-A involves: development of an individualised version of the Clark and Wells (1995) model; an experiential exercise to help patients discover the unhelpful effects of selffocused attention and safety behaviours; video feedback; systematic training in externally focused, non-evaluative attention; and behavioural experiments. When indicated, there may also be work on: socially traumatic memories; anticipatory worry and post-event processing; low self-esteem; parental beliefs and behaviours; and bullying and its consequences. Further details of the treatment with case examples are described by Leigh and Clark (2016) and can also be found at: www.oxcadatresources.com

\section{Training}

The training employed a two-stage approach (Beidas and Kendall, 2010; Rakovshik and McManus, 2010) involving an initial workshop followed by a period of supervised practice. The 2-day workshop involved a combination of teaching, video material, and experiential exercises and role-plays. Clinicians were provided with a detailed treatment manual and a memory stick containing extended training videos ahead of the workshop (these resources are available to access at: www.oxcadatresources.com). After the workshop, clinicians attended weekly supervision via video-link or telephone in small groups. Supervision sessions involved detailed discussion of the previous treatment session, review of questionnaire measures, discussion of any problems that had arisen, and then step-by-step planning for the next session. Videotaped treatment sessions were discussed on a regular basis in supervision.

\section{Setting and therapists}

Two NHS Foundation Trusts in England participated: Oxford Health and Berkshire Healthcare NHS Foundation Trusts. Seven therapists (four from Oxford Health) completed the training: three clinical psychologists and three CBT therapist. All had at least 2 years of experience using CBT and of working with young people. One therapist attended the initial training workshop and three supervision sessions but then withdrew from the study due to a change in circumstances, therefore $n=6$ therapists for the report of clinical outcomes and $n=7$ therapists for cost outcomes.

\section{Inclusion and exclusion criteria for adolescent patients}

Young people (11-17.5 years) with a primary DSM-5 diagnosis of social anxiety disorder routinely referred to CAMHS were eligible. If young people were prescribed psychotropic medication the dosage must have been stable for 2 months. Young people with established autistic spectrum disorders, learning disabilities, suicidal intent or recurrent self-harm, or identified by social services as currently 'at risk' due to child protection concerns were not eligible. 


\section{Outcome measures}

The Anxiety Disorders Interview Schedule (ADIS) - child and parent report (Silverman and Albano, 1996) was administered at baseline and follow-up to young people and parents by psychology postgraduates trained to a high level of inter-rater reliability [kappa/intraclass correlation was $>.85$ for diagnoses and Clinical Severity Ratings (CSR) across all anxiety disorders and for SAD specifically]. For each disorder, a CSR was assigned (from 0 to 8), with scores of 4 or more indicating presence of a disorder.

The self-report version of the Liebowitz Social Anxiety Scale for Children and Adolescents (LSAS-CA-SR; Masia-Warner et al., 1999) assesses social anxiety. Fear and avoidance of 24 social situations are rated on a 4-point scale (total range 0-144). It has well-established psychometric properties (Masia-Warner et al., 2003).

The Social Participation and Satisfaction Questionnaire (Alden and Taylor, 2011) assesses participation in and satisfaction with relationships.

Concentration in class was assessed by asking young people to rate their ability to concentrate in class in the preceding week (0-100) (Leigh and Clark, 2016).

Symptoms of anxiety and depression were assessed using the child version of the Revised Child Anxiety and Depression Scale (RCADS; Chorpita et al., 2000). It has robust psychometric properties (Chorpita et al., 2005).

\section{Process measures}

Several measures covering central processes in cognitive models of social anxiety (Clark et al., 2003) adapted for use with adolescents (Leigh and Clark, 2016) were used to guide therapy.

The Child \& Adolescent Social Summary Weekly Rating Scale (CASSWRS; Clark et al., 2003) was used as an additional measure of social anxiety, obtaining an average score (range $0-8$ ).

The Child \& Adolescent Social Cognitions Questionnaire (CASCQ) covers 22 common social anxiety-related negative thoughts. A mean belief rating is obtained (range 0-100).

The Child \& Adolescent Social Attitudes Questionnaire (CASAQ) is a 41-item scale measuring social anxiety-related beliefs. A mean belief rating is obtained (range 1-7).

The Child \& Adolescent Social Behaviour Questionnaire (CASBQ) is a 29-item scale of common social safety-seeking behaviours. A mean score is obtained (range 0-3).

All of these measures can be obtained from: www.oxcadatresources.com

\section{Treatment acceptability rating}

At the end of treatment, participants rated how acceptable they found the treatment (two items rated on a 5-point scale: 'I was satisfied with the treatment' and 'I would recommend the treatment to others').

\section{Health economic records}

Clinician logs (clinician includes both supervisors and therapists) were designed to capture the amount of healthcare resources (i.e. qualified staff time) necessary to implement CT-SAD-A. They were completed by both supervisors and therapists, who recorded the amount of time spent in activities related to the CT-SAD-A treatment, including training, supervision, preparation and delivery of CT-SAD-A. Data recorded in the clinician logs were used to calculate the total mean amount of qualified staff time used by the NHS per adolescent treated. Results were stratified by type of staff time use. 


\section{Analysis}

Comparisons between pre-treatment and follow-up were made with non-parametric Wilcoxon signed ranks tests. The $Z$-statistic is presented, with associated $p$-values and effect size estimates $(r)$. An effect size of $\geq 0.1$ is interpreted as small; $\geq 0.3$ medium; and $\geq 0.5$ is large (Cohen, 1988). Reliable change on our primary outcome measure, the LSAS-CA-SR, was defined as a pre-follow-up change exceeding the measurement error of the scale (Evans et al., 1998), based on data from Masia-Warner et al. (2003), equating to a change of 16.13 or more. Reliable and clinically significant improvement (RCSI) was defined as pre-follow-up change of at least 2 standard deviations (SD) from the original mean (Evans et al., 1998), equating to a drop of at least 48.75 points.

In the cost analyses, for each study participant, all components of treatment costs, stratified by category of resource use, were computed by multiplying units of resource use by their unit costs (Curtis, 2017) (see Supplementary material, Table S1), expressed in 2016/17 UK pounds sterling $(\mathfrak{E})$. Mean imputation was used to handle missing data, as they referred to the time spent on faceto-face therapist contact with the young person, and were highly deterministic (i.e. readily identifiable and standardised given observed practice). A conditional imputation method was conducted whereby missing data were estimated as an average of known durations for the specific patient. Results were reported in terms of total amount of qualified staff time and associated NHS costs for training and supervision as well as in terms of mean values of qualified staff time and associated NHS cost per patient treated, with variability around the mean measured by standard deviations.

\section{Results}

\section{Characteristics of patients}

The ten females and two males had an average age of 15.17 years $(\mathrm{SD}=1.80$, range $12-17)$. The sample was predominantly White British (10/12), which was representative of the local areas. None reported being prescribed psychotropic medication. All met DSM-5 criteria for SAD. Only two patients had no co-morbid diagnoses; GAD was present in eight patients, and depression (either major depressive disorder or dysthymia) in three. There were five diagnoses of specific phobia pre-treatment across four patients, with one patient diagnosed with two specific phobias. One patient had a diagnosis of post-traumatic stress disorder and one had a diagnosis of panic disorder.

Seven patients received the planned 14 weekly therapy sessions, two patients received 10 weekly sessions, and one patient each received 11 sessions, 12 sessions and 17 sessions. Seven patients received at least one booster session. The follow-up assessment was carried out on average 2.88 months $(S D=1.15$, range $1.16-4.63)$ after the final weekly therapy session.

\section{Outcomes}

\section{Social anxiety symptoms}

Table 1 presents baseline characteristics and social anxiety symptom outcomes at post-treatment and follow-up for patients. Treatment was associated with a significant reduction in social anxiety symptoms on the LSAS-CA-SR at follow-up $(Z=-3.06, p=.002, r=0.62)$. Scores dropped on average $66.80 \%$ over time (range $3-100 \%$; Fig. 1 ) and ten participants, $83 \%$ of the sample, made a reliable improvement. Nine of these participants also demonstrated a reliable and clinically significant improvement. RCADS social anxiety T-scores showed a significant and large decrease over treatment $(Z=-2.93, p=.003, r=0.60)$ : eight patients were in the borderline or clinical range at pretreatment, based on age and gender (T-score $>65$ ), whereas only one patient remained in the clinical range at follow-up. 
Table 1. Social anxiety symptom outcome measures

\begin{tabular}{|c|c|c|c|c|c|c|c|c|c|}
\hline \multirow{2}{*}{$\begin{array}{l}\text { Client } \\
\text { (therapist) }\end{array}$} & \multirow{2}{*}{$\begin{array}{c}\text { Weekly } \\
\text { sessions }(n)\end{array}$} & \multicolumn{3}{|c|}{ LSAS-CA-SR } & \multicolumn{2}{|c|}{ ADIS SAD CSR } & \multicolumn{3}{|c|}{ RCADS Social Anxiety } \\
\hline & & Pre & Post & FU & Pre & FU & Pre & Post & FU \\
\hline $1(1)$ & 14 & 77 & 36 & 21 & 6 & 0 & 72.39 & 44.28 & 44.28 \\
\hline $2(1)$ & 14 & 123 & 40 & 28 & 6 & 0 & 81.92 & 46.28 & 46.28 \\
\hline $3(2)$ & 14 & 109 & 51 & 34 & 7 & 4 & 58.08 & 35.81 & 31.76 \\
\hline $4(2)$ & 14 & 105 & 49 & 20 & 6 & 3 & 59.46 & 39.46 & 39.46 \\
\hline $5(3)$ & 14 & 126 & 41 & 32 & 7 & 5 & 138.65 & 42.27 & 46.29 \\
\hline $6(3)$ & 14 & 87 & 2 & 0 & 4 & 0 & 68.20 & 25.69 & 29.74 \\
\hline $7(4)$ & 10 & 53 & 0 & 0 & 5 & 0 & 58.33 & 26.69 & 26.69 \\
\hline $8(4)$ & 12 & 94 & 5 & 8 & 7 & 0 & 74.40 & 30.22 & 30.22 \\
\hline $9(5)$ & 11 & 57 & 5 & 2 & 5 & 0 & 59.46 & 33.46 & 35.46 \\
\hline $10(5)$ & 14 & 137 & 65 & 103 & 7 & 8 & 74.40 & 44.28 & 58.08 \\
\hline $11^{*}(6)$ & 10 & 100 & 97 & 97 & 7 & $x$ & 79.46 & 79.46 & 79.46 \\
\hline $12(6)$ & 17 & 100 & 103 & 89 & 7 & 7 & 66.37 & 56.33 & 60.34 \\
\hline Median & & 100.09 & 40.50 & 24.50 & 6.50 & 0.00 & 70.30 & 40.87 & 41.89 \\
\hline$[\mathrm{Q} 1, \mathrm{Q} 3]$ & & $\begin{array}{l}\text { [79.50, } \\
119.50]\end{array}$ & $\begin{array}{l}{[5.00} \\
61.50]\end{array}$ & $\begin{array}{l}\text { [3.50, } \\
75.25]\end{array}$ & $\begin{array}{l}{[5.25,} \\
7.00]\end{array}$ & $\begin{array}{l}{[0.00,} \\
5.00]\end{array}$ & $\begin{array}{l}\text { [59.46, } \\
78.19]\end{array}$ & $\begin{array}{c}{[31.03,} \\
45.78]\end{array}$ & $\begin{array}{l}{[30.61,} \\
55.13]\end{array}$ \\
\hline Mean & & 97.41 & 41.17 & 36.18 & 6.17 & 2.45 & 74.26 & 42.02 & 44.00 \\
\hline$[S D]$ & & [26.02] & [35.08] & [38.31] & [1.03] & [3.11] & [21.93] & [14.75] & [15.58] \\
\hline
\end{tabular}

Pre, pre-treatment assessment; Post, post-treatment assessment; FU, follow-up assessment; LSAS-CA-SR, Liebowitz Social Anxiety Scale Child \& Adolescent - Self-Report Version; ADIS SAD CSR, Anxiety Disorders Interview Schedule Social Anxiety Disorder Clinical Severity Rating; RCADS, Revised Children's Anxiety \& Depression Scale; SD, standard deviation; Q, quartile.*Participant 11 did not attend the follow-up assessment. Scores from their final weekly appointment are presented and included in follow-up summary statistics. $x$ indicates missing data.

\section{Diagnostic outcomes ${ }^{1}$}

Seven patients (64\%) lost their primary diagnosis of SAD (see Table 1). Six lost their diagnosis of $\mathrm{GAD}$, and three diagnoses of specific phobia were lost (one patient had two phobias at pretreatment and lost one of these at follow-up). Depressive disorders increased from three to four by follow-up. Of the four patients who retained their diagnosis of SAD at follow-up, one had lost their diagnosis of depression, two had retained their diagnosis of depression, and one had acquired a new diagnosis of depression. One patient lost their diagnosis of SAD but had become depressed at follow-up. Six patients (55\%) were free of all diagnoses at follow-up.

\section{Other clinical outcomes}

As can be seen in Table 2, there was a significant drop in RCADS depression subscale scores ${ }^{1}$ over time $(Z=-2.94, p=.003, r=0.63)$. For $10 / 11$ patients, depression scores were below threshold $(\mathrm{T}<65)$ at follow-up (whilst $5 / 11$ had been below threshold pre-treatment). ID4 continued to show elevated symptoms of depression at follow-up, and also developed a new diagnosis of depression over the course of treatment, whilst losing their SAD diagnosis (see diagnostic outcomes section). RCADS depression score for the other patient (ID12) who had been assigned a new diagnosis of depression during the course of treatment was above threshold at the start of treatment and showed a small decrease at follow-up.

There was a large and significant drop in RCADS GAD scores ${ }^{1}$ over the course of treatment, with all patients scoring below threshold at follow-up $(Z=-3.02, p=.003, r=0.64)$. The group as a whole showed large improvements in social participation $(Z=-2.90, p=.004$,

\footnotetext{
${ }^{1}$ Follow-up diagnostic, RCADS Depression and RCADS GAD data were missing for ID11 and therefore $n=11$ for these results. ID11 had a co-morbid diagnosis of GAD at baseline assessment.
} 


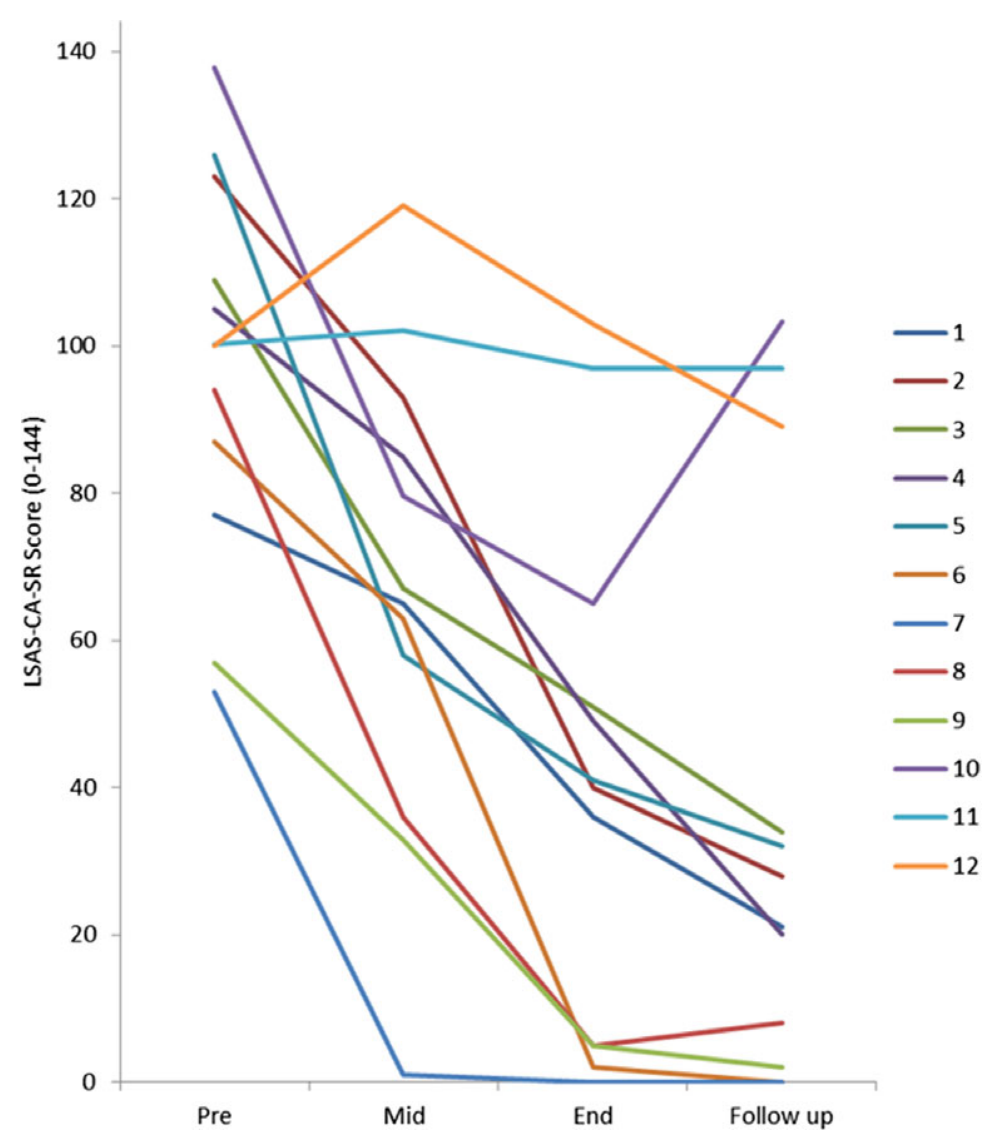

Figure 1. Individual patient LSAS-CA-SR scores at the following measurement points: pre-assessment, mid-treatment, at the end of weekly sessions, and at the follow-up assessment.

$r=0.59)$ and moderate improvements at trend level in social satisfaction $(Z=-1.96$, $p=.050, r=0.40)$.

The group showed a small to medium improvement in concentration $(r=0.30)$, although this was non-significant $(Z=-1.49, p=.137)$. As can be seen in Table 2 , one young person showed no change (ID10) and three a deterioration in their concentration (ID4, ID11 and ID12). Patients ID10, ID11 and ID12 are those young people who did not show a clinically significant improvement on the LSAS-CA-SR and whilst ID4 lost their diagnosis of SAD, they developed a new diagnosis of depression during treatment.

Table 3 provides descriptive statistics for the process measures. All patients reported some reduction in ratings of belief in social cognitions and the group as a whole showed a large reduction in belief ratings on the ASCQ $(Z=-3.06, p=.002, r=0.62)$. For nine patients these reductions were substantial (belief rating reduced by $88-100 \%$ ), and these same patients also showed a clinically significant improvement on the LSAS-CA-SR. There was a large reduction in safety behaviour use by the end of treatment $(Z=-2.85, p=.004, r=0.58)$, and a large reduction in negative social attitudes (ASAQ) $(Z=-2.67, p=.008, r=0.55)$. Only one patient (ID10) showed a strengthening of their negative social attitudes, and they also retained their SAD diagnosis at follow-up. 
Table 2. Related clinical outcome measures

\begin{tabular}{|c|c|c|c|c|c|c|c|c|c|c|c|}
\hline \multirow{2}{*}{$\begin{array}{l}\text { Client } \\
\text { (therapist) }\end{array}$} & \multirow{2}{*}{$\begin{array}{l}\text { Weekly } \\
\text { sessions } \\
(n)\end{array}$} & \multicolumn{2}{|c|}{$\begin{array}{c}\text { RCADS } \\
\text { Depression }\end{array}$} & \multicolumn{2}{|c|}{ RCADS GAD } & \multicolumn{2}{|c|}{ Concentration } & \multicolumn{2}{|c|}{$\begin{array}{c}\text { Social } \\
\text { participation }\end{array}$} & \multicolumn{2}{|c|}{$\begin{array}{c}\text { Social } \\
\text { satisfaction }\end{array}$} \\
\hline & & Pre & FU & Pre & FU & Pre & FU & Pre & FU & Pre & FU \\
\hline $1(1)$ & 14 & 53.69 & 37.96 & 51.37 & 37.90 & 80 & 95 & 52.00 & 63.00 & 14 & 22 \\
\hline $2(1)$ & 14 & 85.91 & 38.66 & 68.14 & 41.98 & 70 & 80 & 50.00 & 85.00 & 20 & 26 \\
\hline $3(2)$ & 14 & 78.41 & 47.72 & 51.84 & 29.68 & 50 & 50 & 23.00 & 46.00 & 15 & 20 \\
\hline $4(2)$ & 14 & 86.28 & 78.13 & 60.81 & 46.28 & 25 & 5 & 28.00 & 64.00 & 12 & 24 \\
\hline $5(3)$ & 14 & 93.89 & 44.7 & 72.94 & 35.20 & 0 & 40 & 21.00 & 39.19 & 10 & 10 \\
\hline $6(3)$ & 14 & 70.74 & 40.05 & 64.49 & 29.68 & 30 & 70 & 33.00 & 88.00 & 10 & 21 \\
\hline $7(4)$ & 10 & 37.06 & 31.57 & 39.81 & 30.25 & 60 & 100 & 64.00 & 91.00 & 22 & 28 \\
\hline $8(4)$ & 12 & 55.93 & 28.97 & 59.46 & 29.81 & 80 & 100 & 51.00 & 68.00 & 21 & 25 \\
\hline $9(5)$ & 11 & 59.1 & 45.52 & 55.00 & 37.56 & 70 & 80 & 54.00 & 55.00 & 19 & 10 \\
\hline $10(5)$ & 14 & 67.17 & 64.92 & 51.37 & 48.68 & 50 & 80 & 27.00 & 39.00 & 6 & 16 \\
\hline $11^{*}(6)$ & 10 & $x$ & $x$ & $x$ & $x$ & 50 & 40 & 21.00 & 44.00 & 13 & 10 \\
\hline $12(6)$ & 17 & 64.92 & 62.67 & 56.77 & 51.37 & 75 & 60 & 51.00 & 42.00 & 16 & 13 \\
\hline Median & & 67.17 & 44.70 & 56.77 & 37.56 & 55.00 & 65.00 & 41.50 & 59.00 & 14.50 & 20.50 \\
\hline [Q1, Q3] & & $\begin{array}{l}\text { [55.93, } \\
85.91]\end{array}$ & $\begin{array}{l}{[37.96} \\
62.67]\end{array}$ & $\begin{array}{l}{[51.37,} \\
64.49]\end{array}$ & $\begin{array}{l}{[29.81,} \\
46.28]\end{array}$ & $\begin{array}{l}{[35.00} \\
73.75]\end{array}$ & $\begin{array}{l}{[42.40} \\
91.25]\end{array}$ & $\begin{array}{l}{[24.00} \\
51.75]\end{array}$ & $\begin{array}{l}\text { [42.50, } \\
80.75]\end{array}$ & $\begin{array}{l}{[10.50,} \\
19.75]\end{array}$ & $\begin{array}{l}{[10.75,} \\
24.75]\end{array}$ \\
\hline Mean & & 68.46 & 47.35 & 57.46 & 38.04 & 53.33 & 64.17 & 39.58 & 60.35 & 14.83 & 18.75 \\
\hline$[S D]$ & & [16.84] & [15.18] & [9.18] & {$[8.07]$} & {$[24.71]$} & [28.73] & [15.46] & [19.38] & {$[4.97]$} & [6.69] \\
\hline
\end{tabular}

Pre, pre-treatment Assessment; FU, follow-up assessment; RCADS, Revised Children's Anxiety \& Depression Scale; SD, standard deviation; $\mathrm{Q}$, quartile. ${ }^{\star}$ Participant 11 did not attend post-treatment assessment. Scores from their final weekly appointment are presented where available and included in follow-up summary statistics. $x$ indicates missing data.

Table 3. Social anxiety process measures

\begin{tabular}{|c|c|c|c|c|c|c|c|c|c|}
\hline \multirow{2}{*}{$\begin{array}{l}\text { Client } \\
\text { (therapist) }\end{array}$} & \multirow{2}{*}{$\begin{array}{l}\text { Weekly } \\
\text { sessions } \\
(n)\end{array}$} & \multicolumn{2}{|c|}{ CASSWRS } & \multicolumn{2}{|c|}{ CASCQ-B } & \multicolumn{2}{|c|}{ CASAQ } & \multicolumn{2}{|c|}{ CASBQ } \\
\hline & & Pre & FU & Pre & FU & Pre & FU & Pre & FU \\
\hline $1(1)$ & 14 & 4.83 & 1.67 & 43.71 & 0.00 & 4.88 & 2.79 & 1.47 & 0.45 \\
\hline $2(1)$ & 12 & 6.67 & 0.83 & 83.23 & 3.87 & 6.27 & 2.63 & 2.24 & 0.55 \\
\hline $3(2)$ & 14 & 4.67 & 3.83 & 75.00 & 4.16 & 4.81 & 3.00 & 1.70 & 0.55 \\
\hline $4(2)$ & 14 & 5.67 & 0.67 & 70.29 & 2.58 & 4.69 & 2.54 & 1.27 & 0.33 \\
\hline $5(3)$ & 13 & 5.17 & 3.83 & 83.97 & 3.65 & 5.85 & 3.08 & 2.38 & 0.79 \\
\hline $6(3)$ & 14 & 5.67 & 0.50 & 67.10 & 7.67 & 4.87 & 1.21 & 1.79 & 0.24 \\
\hline $7(4)$ & 10 & 1.83 & 0.00 & 3.87 & 0.00 & 2.73 & 1.37 & 0.58 & 0.00 \\
\hline $8(4)$ & 12 & 4.83 & 0.17 & 44.19 & 1.29 & 2.54 & 2.48 & 1.33 & 0.24 \\
\hline $9(5)$ & 11 & 5.17 & 0.00 & 70.32 & 3.45 & 4.31 & 3.21 & 1.18 & 0.36 \\
\hline $10(5)$ & 14 & 6.20 & 5.00 & 84.84 & 61.83 & 2.60 & 3.87 & 1.94 & 2.00 \\
\hline $11^{*}(6)$ & 10 & 6.50 & 6.17 & 52.58 & 40.32 & 5.00 & 4.31 & 1.72 & 1.73 \\
\hline $12(6)$ & 14 & 5.67 & 3.83 & 63.87 & 34.52 & 4.96 & 3.85 & 1.97 & 1.48 \\
\hline Median & - & 5.42 & 1.25 & 68.70 & 3.76 & 4.84 & 2.90 & 1.71 & 0.50 \\
\hline [Q1, Q3] & - & $\begin{array}{c}\text { [4.83, } \\
6.07]\end{array}$ & $\begin{array}{c}\text { [0.25, } \\
3.83]\end{array}$ & $\begin{array}{c}\text { [46.29, } \\
81.17]\end{array}$ & $\begin{array}{c}{[1.61} \\
27.81]\end{array}$ & $\begin{array}{c}\text { [3.13, } \\
4.99]\end{array}$ & $\begin{array}{c}{[2.50} \\
3.69]\end{array}$ & $\begin{array}{c}\text { [1.29, } \\
1.96]\end{array}$ & $\begin{array}{c}\text { [0.26, } \\
1.31]\end{array}$ \\
\hline Mean & - & 5.24 & 2.21 & 61.91 & 13.61 & 4.46 & 2.86 & 1.63 & 0.73 \\
\hline$[S D]$ & - & {$[1.26]$} & {$[2.19]$} & [23.18] & [20.32] & {$[1.22]$} & [0.93] & {$[0.50]$} & [0.65] \\
\hline
\end{tabular}

Pre, pre-treatment assessment; FU, follow-up assessment; CASSWRS, Adolescent Social Summary Weekly Rating Scale; CASCQ-B, Adolescent Social Cognitions Questionnaire Belief Ratings; CASAQ, Adolescent Social Attitudes Questionnaire; CASBQ, Adolescent Social Behaviour Questionnaire. ${ }^{*}$ Participant 11 did not attend their follow-up assessment. Scores from their final weekly appointment are presented and included in the follow-up summary statistics. 


\section{Treatment acceptability}

Eleven young people completed the treatment acceptability scale at the follow-up assessment. All of them agreed (either 'somewhat' or 'completely') with the statements 'I was satisfied with the treatment' and 'I would recommend the treatment to others'.

\section{Cost of treatment delivery in NHS CAMHS}

Mean supervisor time per therapist attending each workshop was 278 minutes $(S D=157)$ and

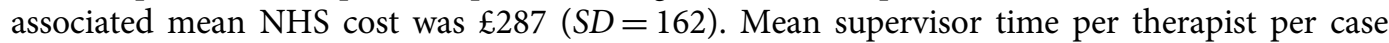
supervision week was $50 \mathrm{~min}(S D=14)$ and associated mean NHS cost was $£ 51.50$ $(S D=14.6)$ (Supplementary material, Table S2). Mean therapist time for each workshop attended was $420 \mathrm{~min}(7 \mathrm{~h})(S D=0)$ and associated mean NHS cost was $£ 294(S D=0)$. On average, each therapist participated in $24(S D=12)$ case supervision sessions. Mean therapist time per case supervision session attended was $73 \mathrm{~min}(S D=40)$ and associated mean NHS cost was $\mathfrak{E 5 1}(S D=28)$ (Supplementary material, Table $S 3)$. Each adolescent treated received on average $5279 \mathrm{~min}$ (around $88 \mathrm{~h})(S D=1301)$ of qualified clinician time (including therapist's training and case supervision) and this would cost the NHS $£ 4047(S D=1003)$ per adolescent treated. The main cost driver was face-to-face delivery of the CT-SAD treatment ( $1861 ; S D=358$ per adolescent treated) (Supplementary material, Table S4).

\section{Discussion}

The findings of the training case series provide preliminary evidence that good outcomes can be achieved from the delivery of CT-SAD-A in standard NHS CAMHS during supervised practice. Patients were routine referrals to CAMHS services. All patients presented with a primary diagnosis of SAD and received CT-SAD-A provided by CAMHS therapists as part of their training in this novel treatment. The group as a whole demonstrated large and significant improvements in social anxiety symptoms, as well as associated clinical outcomes. Substantial improvements in the proposed psychological mechanisms that are proposed to drive change in this treatment (social beliefs and behaviours) were also reported and treatment acceptability was high. To the best of our knowledge, this is the first detailed cost analysis of treatment for $\mathrm{SAD}$ in young people. The economic analysis indicated that the total cost to the NHS of the CT-SAD-A treatment was $£ 4047$ ( $S D=£ 1003$ ) per adolescent treated, of which $£ 1861$

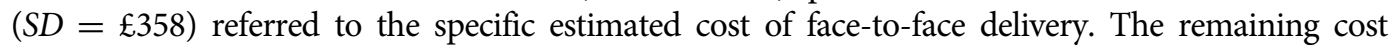
was for training and supervising therapists who were not previously familiar with the treatment.

As in the first case series of CT-SAD-A, undertaken by our group (Leigh and Clark, 2016), the drop in LSAS was substantial (67\%) at follow-up. The mean change of 61 points on the LSAS reported here compares favourably with the figures reported for adult CT-SAD; e.g. mean changes of 43 and 46 on the LSAS reported by Clark and colleagues (Clark et al., 2003 and Clark et al., 2006, respectively). From visual inspection of the LSAS-CA-SR (see Fig. 1), it is clear that nine of the 12 patients reported considerable improvement in symptoms. They reported an average reduction of $85 \%$ on the measure ( $v s 13 \%$ for the other three patients) and attained clinically significant improvement. Seven of the nine patients lost their primary SAD diagnosis and the other two showed reductions on their ADIS CSR SAD rating (ID3: 7 to 4; ID5: 7 to 5). All nine patients improved to below clinical thresholds on the social anxiety and GAD subscales of RCADS. These findings, and our loss of diagnosis rate of $64 \%$ for the sample overall, compare favourably with outcomes of children and young people with SAD from trials of more general forms of CBT. For example, Ginsburg et al. (2011) reported recovery rates of $40 \%$ among children and young people with SAD who received 14 sessions of a generic form of CBT for anxiety. 
Importantly, scores on measures of the psychological mechanisms that are targeted in CT-SAD-A all reduced substantially over the course of therapy for the nine improvers, in contrast to the non-improvers. On the CASCQ, the average reduction was 96\% (89-100\%) for the nine improvers vs 32\% (23-46\%) for the three non-improvers; on the CASBQ it was $77 \%(68-100 \%)$ vs $7 \%(-3-25 \%)$; and on the CASAQ it was $43 \%(2-75 \%) v s-4 \%(-48-22 \%)$.

Whilst we would not expect any treatment to be effective for all patients, it is interesting to consider why the three remaining patients responded considerably less well to therapy compared with the other nine. As can be seen from Fig. 1, it does not appear to be a function of SAD severity. Possible explanations include factors relating to how the treatment was delivered. It may be that the particular therapists for these patients did not gain as much from the training as the others, or had less time available, so had less time to prepare, were less able to conduct full length sessions $(90 \mathrm{~min}$ ), and as such were less able to facilitate in vivo behavioural experiments. Alternatively, it might be that differences in therapist allegiance affected uptake and delivery of CT-SAD-A techniques. In future, examination of therapist allegiance ratings and blind rating of therapist competency could shed light on these hypotheses. Larger trials would provide the opportunity to examine potential predictors of response to treatment, including therapist and patient-specific factors, more thoroughly and systematically.

Notably, the patient outcomes described are for those treated during the CAMHS therapists' training, and so it is possible that the outcomes reported may be an under-estimate of patients' potential gains and an over-estimate of economic costs, because we would expect therapists to achieve better outcomes as they accrue more experience. Future research would benefit from establishing the extent, and optimum modalities, for delivering therapist training in CT-SAD-A in order to achieve best outcomes. Notwithstanding these issues, the findings highlight the potential benefit of delivering CT-SAD to adolescents.

There are, of course, important limitations of the training case series, including the small sample, which only included two males, and uncontrolled design lacking a baseline assessment phase, which means we cannot rule out the possibility of symptom change due to the passage of time, or non-specific therapy effects. We would suggest that this is unlikely, as the patients had all experienced symptoms for at least 6 months in order to qualify for a diagnosis, and in many cases social anxiety had been present for much longer. We cannot rule out potential non-specific therapeutic benefits of therapist contact and support, and therefore a controlled comparison is needed to test this alternative explanation. Furthermore, longer term outcomes and examination of possible moderators of treatment outcomes should certainly be included in any further systematic evaluation.

Strengths of the study included the thorough assessment and training procedures that were implemented within the CAMHS context. A further strength of our study is the inclusion of a cost analysis, highlighted as lacking by NICE (NICE, 2013). The estimated cost of delivering treatment (excluding training and supervision of therapists) was $£ 1861$ per young person treated within our study, which was admittedly based on a small sample size. Nevertheless, in a tentative comparison with treatments for adults our cost compares favourably to NICE's estimated cost of delivering CT-SAD per patient with adults (£2341) (Mavranezouli et al., 2015), which has been found to be most cost-effective when psychological and pharmacological interventions for SAD adults have been compared (NICE, 2013).

Our economic results need to be interpreted in light of some limitations. While the response rate of supervisor-completed economic logs was $100 \%$ throughout the study duration, therapistcompleted economics logs had rates of missing values that ranged from 50 to $67 \%$ for various treatment session across all patients, and imputation methods had to be used to handle missing data. Using mean imputation reduced sample variability with the consequence that uncertainty around our cost estimates was under-estimated. It should also be noted that the way in which the training phase was conducted was less than optimal due to the fact that new 
therapists were recruited at different times throughout the training programme. Consequently, two cohorts of therapists were trained separately, causing a duplication of supervisors' time in the training phase which led to inflated costs. In 'real world' practice, workshops could be delivered to much larger numbers of therapists. This should be examined in future studies. Finally, the lack of a control condition precludes any conclusions about cost-effectiveness (i.e. whether CT-SAD-A may be good value for money) in comparison with current usual practice.

Our preliminary findings suggest that therapists working in busy CAMHS settings can achieve good outcomes for routinely referred SAD patients even whilst undergoing their training in the therapy and our results point to the potential value of delivering the training more widely. Based on our small sample and working within the current CAMHS context, the cost of delivering CT-SAD-A with adolescents appeared to be no more than the cost of delivering CT-SAD with adults, and would be expected to bring greater economic advantages due to the earlier onset of benefits, including potentially related to increased engagement in education.

Supplementary material. To view supplementary material for this article, please visit: https://doi.org/10.1017/S1352465 821000035

Data availability. The data that support the findings of this study are available from the corresponding author (E.L.), upon reasonable request.

Acknowledgements. We would like to express wholehearted thanks to the participating families, therapists, their service managers and clinical leads, and the R\&D teams at the Oxford Health and Berkshire Healthcare NHS Foundation Trusts. We are also grateful for the comments of the other members of the Trial Management Group.

Financial support. This work was supported by the National Institute of Health Research (NIHR HTA 14/170/01; NIHR Research Professorship; C. Creswell; Senior Investigator (D.M. Clark)); Wellcome Trust (Clinic Research Fellowship (E. Leigh); Programme Grant (D.M. Clark). M.V. receives funding from the National Institute for Health Research (NIHR) Applied Research Collaboration Oxford and Thames Valley at Oxford Health NHS Foundation Trust, and the NIHR Oxford Biomedical Research Centre. The views expressed are those of the author(s) and not necessarily those of the NHS, the NIHR or the Department of Health and Social Care.

Ethics statement. All authors have abided by the Ethical Principles of Psychologists and code of Conduct as set out by the BABCP and BPS. Ethical approval for this study was gained from the local NHS Research Ethics Committee (REC reference: 16/SC/0315).

Conflicts of interest. David M. Clark is one of the authors of the cognitive model of social anxiety that CT-SAD-A is based on and has also played a central role in developing CT-SAD. No other conflicts of interest are declared.

\section{References}

Alden, L. E., \& Taylor, C. T. (2011). Relational treatment strategies increase social approach behaviors in patients with generalized social anxiety disorder. Journal of Anxiety Disorders, 25, 309-318.

Beesdo, K., Pine, D. S., Lieb, R., \& Wittchen, H. (2010). Incidence and risk patterns of anxiety and depressive disorders and categorization of generalized anxiety disorder. Archives of General Psychiatry, 67, 47-57. doi: 10.1001/archgenpsychiatry. 2009.177

Beidas, R. S., \& Kendall, P. C. (2010). Training therapists in evidence-based practice: a critical review of studies from a systems-contextual perspective. Clinical Psychology: Science and Practice, 17, 1-30. doi: 10.1111/j.1468-2850.2009. 01187.x

Bruce, S. E., Yonkers, K. A., Otto, M. W., Eisen, J. L., Weisberg, R. B., Pagano, M., . . \& Keller, M. B. (2005). Influence of psychiatric comorbidity on recovery and recurrence in generalized anxiety disorder, social phobia, and panic disorder: a 12-year prospective study. American Journal of Psychiatry, 162, 1179-1187. doi: 10.1176/appi.ajp.162.6.1179

Chorpita, B. F., Moffitt, C. E., \& Gray, J. (2005). Psychometric properties of the Revised Child Anxiety and Depression Scale in a clinical sample. Behaviour Research \& Therapy, 43, 309-322. doi: 10.1016/j.brat.2004.02.004

Chorpita, B. F., Yim, L., Moffitt, C., Umemoto, L. A., \& Francis, S. E. (2000). Assessment of symptoms of DSM-IV anxiety and depression in children: a revised child anxiety and depression scale. Behaviour Research and Therapy, 38, 835-855. doi: 10.1016/j.brat.2004.02.004 
Clark, D. M., Ehlers, A., Hackmann, A., McManus, F., Fennell, M., Grey, N., . . \& Wild, J. (2006). Cognitive therapy versus exposure and applied relaxation in social phobia: a randomized controlled trial. Journal of Consulting and Clinical Psychology, 74, 568-578. doi: 10.1037/0022-006x.74.3.568

Clark, D. M., Ehlers, A., McManus, F., Hackmann, A., Fennell, M., Campbell, H., ... \& Louis, B. (2003). Cognitive therapy versus fluoxetine in generalized social phobia: a randomized placebo-controlled trial. Journal of Consulting and Clinical Psychology, 71, 1058-1067. doi: 10.1037/0022-006x.71.6.1058

Clark, D. M., \& Wells, A. (1995). A cognitive model of social phobia. In G. Heimberg, M. R. M. R. Liebowitz, D. Hope, \& F. Scheier (eds), Social Phobia: Diagnosis, Assessment, and Treatment (pp. 69-93). New York, USA: Guilford Press.

Cohen, J. (1988). Statistical Power Analysis for the Behavioral Sciences (2nd edn). Hillsdale, NJ, USA: Erlbaum.

Creswell, C., Waite, P. \& Hudson, J. (2020). Anxiety disorders in children and young people: assessment and treatment. Journal of Child Psychology and Psychiatry, 61, 628-643. doi: 10.1111/jcpp.13186

Curtis L. A. (2017). Unit costs of health and social care. University of Kent, 2017; https://kar.kent.ac.uk/65559/ (accessed 7 February 2019).

Evans, C., Margison, F., \& Barkham, M. (1998). The contribution of reliable and clinically significant change methods to evidence-based mental health. Evidence-Based Mental Health, 1, 70-72. doi: 10.1136/ebmh.1.3.70

Ginsburg, G. S., Kendall, P. C., Sakolsky, D., Compton, S. N., Piacentini, J., Albano, A. M., ... \& March, J. (2011). Remission after acute treatment in children and adolescents with anxiety disorders: findings from the CAMS. Journal of Consulting and Clinical Psychology, 79, 806-813. doi: 10.1037/a0025933

Ginzburg, D. M., Bohn, C., Höfling, V., Weck, F., Clark, D. M., \& Stangier, U. (2012). Treatment specific competence predicts outcome in cognitive therapy for social anxiety disorder. Behaviour Research and Therapy, 50, 747-752. doi: 10.1016/j.brat.2012.09.001

Hodson, K. J., McManus, F. V., Clark, D. M., \& Doll, H. (2008). Can Clark and Wells' (1995) cognitive model of social phobia be applied to young people? Behavioural and Cognitive Psychotherapy, 36, 449-461.

Ingul, J. M., Aune, T., \& Nordahl, H. M. (2014). A randomized controlled trial of individual cognitive therapy, group cognitive behaviour therapy and attentional placebo for adolescent social phobia. Psychotherapy and Psychosomatics, 83, 54-61. doi: 10.1159/000354672

Kessler, R. C., Berglund, P., Demler, O., Jin, R., Merikangas, K. R., \& Walters, E. E. (2005). Lifetime prevalence and age-ofonset distributions of DSM-IV disorders in the National Comorbidity Survey Replication. Archives of General Psychiatry, 62, 593-602. doi: 10.1001/archpsyc.62.6.593

La Greca, A. M., \& Harrison, H. M. (2005). Adolescent peer relations, friendships, and romantic relationships: do they predict social anxiety and depression? Journal of Clinical Child and Adolescent Psychology, 34, 49-61. doi: 10.1207/ s15374424jccp3401_5

Leichsenring, F., Salzer, S., Beutel, M. E., Herpertz, S., Hiller, W., Hoyer, J., . . \& Leibing, E. (2013). Psychodynamic therapy and cognitive-behavioral therapy in social anxiety disorder: a multicenter randomized controlled trial. American Journal of Psychiatry, 170, 759-767. doi: 10.1176/appi.ajp.2013.12081125

Leigh, E., \& Clark, D. M. (2016). Cognitive therapy for social anxiety disorder in adolescents: a development case series. Behavioural and Cognitive Psychotherapy, 44, 1-17. doi: 10.1017/s1352465815000715

Masia-Warner, C., Klein, R. G., \& Liebowitz, M. R. (1999). The Liebowitz Social Anxiety Scale for Children and Adolescents (LSAS-CA). Available from Carrie Masia-Warner, Dickson Hall 255, 1 Normal Avenue, Montclair, NJ, USA 07043.

Masia-Warner, C., Storch, E. A., Pincus, D. B., Klein, R. G., Heimberg, R. G., \& Liebowitz, M. R. (2003). The Liebowitz Social Anxiety Scale for Children and Adolescents: an initial psychometric investigation. Journal of the American Academy of Child \& Adolescent Psychiatry, 42, 1076-1084. doi: 10.1097/01.CHI.0000070249.24125.89

Mavranezouli, I., Mayo-Wilson, E., Dias, S., Kew, K., Clark, D. M., Ades, A., \& Pilling, S. (2015). The cost effectiveness of psychological and pharmacological interventions for social anxiety disorder: a model-based economic analysis. PLoS One, 10, e0140704.

Mayo-Wilson, E., Dias, S., Mavranezouli, I., Kew, K., Clark, D. M., Ades, A., \& Pilling, S. (2014). Psychological and pharmacological interventions for social anxiety disorder in adults: a systematic review and network meta-analysis. The Lancet Psychiatry, 1, 368-376. doi: 10.1016/S2215-0366(14)70329-3.

Merikangas, K. R., Nakamura, E. F., \& Kessler, R. C. (2009). Epidemiology of mental disorders in children and adolescents. Dialogues in Clinical Neuroscience, 11, 7-20.

Mörtberg, E., Clark, D. M., Sundin, Ö., \& Åberg Wistedt, A. (2007). Intensive group cognitive treatment and individual cognitive therapy vs. treatment as usual in social phobia: a randomized controlled trial. Acta Psychiatrica Scandinavica, 115, 142-154. doi: 10.1111/j.1600-0447.2006.00839.x

NICE (2013). National Institute for Health and Care Excellence: Social anxiety disorder: recognition, assessment and treatment 2013; https://wwwniceorguk/guidance/cg159 (accessed 7 February 2019).

Rakovshik, S. G., \& McManus, F. (2010). Establishing evidence-based training in cognitive behavioral therapy: a review of current empirical findings and theoretical guidance. Clinical Psychology Review, 30, 496-516. doi: 10.1016/j. cpr.2010.03.004 
Ranta, K., Kaltiala-Heino, R., Rantanen, P., \& Marttunen, M. (2009). Social phobia in Finnish general adolescent population: prevalence, comorbidity, individual and family correlates, and service use. Depression and Anxiety, 26, 528-536. doi: 10.1002/da.20422

Rapee, R. M., \& Heimberg, R. G. (1997). A cognitive-behavioral model of anxiety in social phobia. Behaviour Research and Therapy, 35, 741-756. doi: 10.1016/S0005-7967(97)00022-3

Schreiber, F., \& Steil, R. (2013). Haunting self-images? The role of negative self-images in adolescent social anxiety disorder. Journal of Behavior Therapy and Experimental Psychiatry, 44, 158-164.

Silverman, W. K., \& Albano, A. M. (1996). The Anxiety Disorders Interview Schedule for DSM-IV: Child and Parent Versions. San Antonio, TX, USA: Graywind Publications, A Division of The Psychological Corporation.

Stangier, U., Heidenreich, T., Peitz, M., Lauterbach, W., \& Clark, D. M. (2003). Cognitive therapy for social phobia: individual versus group treatment. Behaviour Research \& Therapy, 41, 991-1007. doi: 10.1016/S0005-7967(02) 00176-6

Stangier, U., Schramm, E., Heidenreich, T., Berger, M., \& Clark, D. M. (2011). Cognitive therapy vs interpersonal psychotherapy in social anxiety disorder: a randomized controlled trial. Archives of General Psychiatry, 68, 692-700. doi: 10.1001/archgenpsychiatry.2011.67

Stein, M. B., \& Kean, Y. M. (2000). Disability and quality of life in social phobia: epidemiologic findings. American Journal of Psychiatry, 157, 1606-1613. doi: 10.1176/appi.ajp.157.10.1606

Wittchen, H.-U., Fuetsch, M., Sonntag, H., Müller, N., \& Liebowitz, M. (1999). Disability and quality of life in pure and comorbid social phobia - findings from a controlled study. European Psychiatry, 14, 118-131. doi: 10.1016/S0924-9338(00) 00211-X

Cite this article: Leigh E, Creswell C, Stallard P, Waite P, Violato M, Pearcey S, Brooks E, Taylor L, Warnock-Parkes E, and Clark DM (2021). Delivering cognitive therapy for adolescent social anxiety disorder in NHS CAMHS: a clinical and cost analysis. Behavioural and Cognitive Psychotherapy 49, 385-397. https://doi.org/10.1017/S1352465821000035 